\title{
Total-Variation Minimization on Unstructured Volumetric Mesh: Biophysical Applications on Reconstruction of 3D Ischemic Myocardium
}

\author{
Jingjia Xu*, Azar Rahimi Dehaghani*, Fei Gao ${ }^{\dagger}$, and Linwei Wang* \\ * Golisano College of Computing and Information Sciences, Rochester Institute of Technology, Rochester, NY, USA. \\ $\dagger$ Molecular Imaging Division, Siemens Medical Solutions, Knoxville, TN, USA.
}

\begin{abstract}
This paper describes the development and application of a new approach to total-variation (TV) minimization for reconstruction problems on geometrically-complex and unstructured volumetric mesh. The driving application of this study is the reconstruction of $3 \mathrm{D}$ ischemic regions in the heart from noninvasive body-surface potential data, where the use of a TV-prior can be expected to promote the reconstruction of two piecewise smooth regions of healthy and ischemic electrical properties with localized gradient in between. Compared to TV minimization on regular grids of pixels/voxels, the complex unstructured volumetric mesh of the heart poses unique challenges including the impact of mesh resolutions on the TVprior and the difficulty of gradient calculation. In this paper, we introduce a variational TV-prior and, when combined with the iteratively re-weighted least-square concept, a new algorithm to TV minimization that is computationally efficient and robust to the discretization resolution. In a large set of simulation studies as well as two initial real-data studies, we show that the use of the proposed TV prior outperforms L2-based penalties in reconstruct ischemic regions, and it shows higher robustness and efficiency compared to the commonly used discrete TV prior. We also investigate the performance of the proposed TVprior in combination with a L2- versus L1-based data fidelity term. The proposed method can extend TV-minimization to a border range of applications that involves physical domains of complex shape and unstructured volumetric mesh.
\end{abstract}

\section{INTRODUCTION}

Myocardial ischemia, a precursor of myocardial infarction, occurs when the oxygen supply to the heart is insufficient. Myocardial infarction creates substrate for ventricular arrhythmias and increase the risk of sudden cardiac death [8]. Nowadays, 12-lead electrocardiograms (ECG) is routinely used for monitoring and identifying myocardial ischemia [22], although its low sensitivity and inability to locate ischemic region has been noted in [1]. Some tomographic techniques can also be used for ischemia imaging, such as perfusion scintigraphy [1]. However, high cost and long procedure time limit the wide application of such techniques in the clinic [9].

Alternatively, much effort has been devoted to computational approaches that reconstruct ischemic regions from body-surface ECG data, mostly through the reconstruction of potential sequence on the epicardium [2]. These approaches define location and extent of ischemic regions on the heart surface only, without information on the transmurality or 3D morphology of ischemic myocardium. Transition to 3D reconstruction of ischemic regions has been difficult because this problem is not only mathematically ill-posed due to the limited number of measurements, but also-as determined by the biophysics of the underlying electromagnetic field-has non-unique physical solutions [14]. Existing methods can generally be divided into two categories. One type of approaches subjects the reconstruction to physiological constraints enforced by computational models of cardiac excitation throughout the entire ECG cycle, either through deterministic optimization [10] or probabilistic estimation [20]. While the incorporation of spatiotemporal physiological constraints help overcome the ill-posedness of the reconstruction problem, it may also impact the accuracy of the reconstruction when the presence of the ischemic region in the heart is unknown a priori. The alternative type of approaches directly regularizes the reconstruction problem with L2 penalty on the solutions, solved by various methods such as the level-set framework [12] or constrained optimization [19].

In this paper, we introduce a TV-prior into the reconstruction of ischemic myocardium using noninvasive ECG data. The ability of TV penalty to promote reconstruction of piecewise homogeneous regions with sharp transitions ideally matches the electrophysiological property of an ischemic heart: during the ST-segment of an ECG cycle, action potential of healthy myocardium will be mostly uniform and its spatial gradient is expected to be close to zero. In a ischemic heart, the potential difference between ischemic and healthy myocardium will create a steep gradient along the ischemic region (Fig. $1(a, b)$ ). The application of TV minimization, therefore, is expected to promote the reconstruction of ischemic region with higher resolution and accuracy along its border.

Since its original development, TV minimization has been widely applied to preserve the sharp edge of an image in a variety of applications, such as blind de-convolution [4] and image de-noising [16]. Most of these existing applications deal exclusively with regular grids of pixels or voxels and, correspondingly, the discrete TV prior is commonly defined as the L1-norm of the gradient field of the grid. In comparison, the biophysical application targeted in this paper involves a complex volumetric mesh of physical meaning (geometry of the heart) discretized with an unstructured grid 


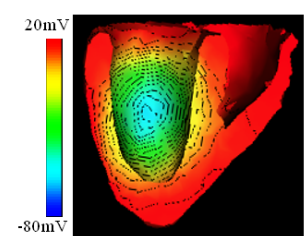

(a) Action potential

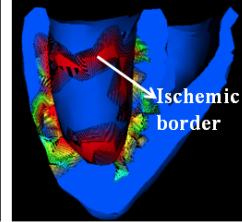

(b) Spatial gradient

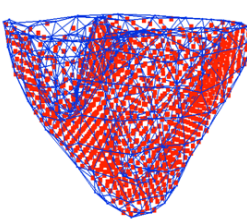

(c) Unstructured mesh
Figure 1. Illustration of 3D action potential (a) and its spatial gradient in an ischemic heart (b); (c) volumetric mesh of the heart

as shown in Fig.1(c). It therefore faces the following unique challenges: 1) the commonly used discrete definition of TV prior - based on the gradient field of the discrete mesh - is highly affected by the resolution of the discrete mesh; and 2) gradient of the discrete mesh - given its complex shape and unstructured grid distribution - is much more difficult and error-prone to calculate than that of a digital image.

This paper is dedicated to developing an approach to overcoming the challenges that arise from TV minimization on a physical domain of complex shape and unstructured mesh discretization. First, to ensure the accuracy of the TV prior and its robustness to the resolution of the discrete mesh, we introduce a variational TV prior that approximates the continuous TV form by a numerical integration with Gaussian quadrature points. Adopting the concept from iteratively re-weighted least-square approximation of L1minimization [15], we then introduce a new iterative algorithm that solves the TV minimization with a sequence of weighted L2 minimization problems, where at each iteration the variational TV-prior is approximated by a weighted, variational quadratic prior. Finally, we investigate the performance of the combination of the TV-prior with a L2versus a L1-based data fidelity term. Through a large set of phantom experiments simulating ischemic myocardium of different locations and sizes within the left ventricle, we successfully demonstrate that: 1) the adoption of TVprior provides significantly higher accuracy over L2-based penalties in localizing action potential gradient along ischemic regions; 2) with the proposed variational TV prior, TV minimization on the irregular cardiac mesh shows higher accuracy, robustness, and computational efficiency compared to that with the commonly used discrete TV prior; and 3) the use of a L1-based data fidelity term provides higher accuracy and convergence rate when the reconstruction faces high measurement noises, while the L2-based data fidelity term provides better performance facing low to medium measurement noises. Real-data experiments on two patients with ischemic hearts further verify the potential of the presented method in reconstructing the steep gradient along the ischemic myocardium border and thus outlining the shape of the ischemic region. Overall, the parented method will help generalize the application of TV minimization extend to problems that involve physical domains of complex shape and unstructured mesh discretization.

\section{Methodology}

First, we briefly introduce the biophysical model underlying the reconstruction problem. Cardiac electrical excitation produces time-varying voltage data that can be easily accessible on the body-surface, as if our torso were a quasistatic electromagnetic field [14]. The spatial distribution of extracellular potential $\phi_{t e}$ within the myocardium $\Omega_{h}$ is governed by the Poisson's equation with cardiac electrical currents as the source term; the distribution of extracellular potential $\phi_{t i}$ within any other organ $\Omega_{t i}$ in the region $\Omega_{t / h}$ bounded by the heart's surface and body surface is determined by Laplace's equation assuming no other active source exist outside the myocardium:

$$
\begin{aligned}
& \sigma_{k} \nabla^{2} \phi_{t e}(\mathbf{r})=\nabla \cdot\left(-\mathbf{D}_{i}(\mathbf{r}) \nabla u(\mathbf{r})\right), \quad \forall \mathbf{r} \in \Omega_{h} ; \\
& \sigma_{t i} \nabla^{2} \phi_{t i}(\mathbf{r})=0, \quad \forall \mathbf{r} \in \Omega_{t i} \cup \Omega_{t i}=\Omega_{t / h}
\end{aligned}
$$

where $\sigma_{k}$ is myocardial conductivity, $\sigma_{t i}$ conductivities of different tissues, $\mathbf{D}_{i}$ intracellular conductivity tensor of the heart, and $\mathbf{r}$ the spatial coordinate.

With different numerical methods, such as finite element method[19] or combined meshfree and boundary element method [21], this biophysical relationship can be solved on a subject-specific heart-torso model derived from tomographic images:

$$
\phi(t)=\mathbf{H u}(\mathbf{t})
$$

where $\phi(t)$ is the voltage data sensed on the body surface, $\mathbf{u}(t)$ the discrete field of transmural action potential across the 3D myocardium, and the transfer matrix $\mathbf{H}$ is specific to each individual's torso anatomy. The condition number of $\mathbf{H}$ was shown at the order of $10^{-14}$ [20].

As explain earlier, we propose to incorporate the TVprior into the reconstruction of $3 \mathrm{D}$ action potentials $\mathbf{u}(t)$ from body-surface measurement $\phi(t)$. At this stage, we exclude the temporal factor and focus on the reconstruction during the ST-segment of the ECG cycle where the above electrical pattern persists in the heart. Mathematically, the reconstruction problem can be formulated as:

$$
\hat{\mathbf{u}}=\min _{\mathbf{u}}\left\{\|\mathbf{H u}-\phi\|_{q}^{q}+\lambda T V(\mathbf{u})\right\}
$$

where $q$ indicate L1 norm or L2 norm of data fidelity term. $T V(\mathbf{u})$ denotes the total-variation of the discrete field of $\mathbf{u}$. For a continuous signal $\mathrm{u}$, its total-variation is defined as [4]: $\int_{\Omega}|\nabla u| d \Omega$. Here we must form a proper discrete definition of $T V(\mathbf{u})$ that is close to its continuous definition regardless of the resolution of the discretization or the complexity of the mesh.

\section{A. Variational TV prior}

In most image-processing applications, a discrete version of $T V(\mathbf{u})$ is calculated as the L1-norm of the discrete 
gradient field of $\mathbf{u}$ :

$T V(\mathbf{u})=\|\nabla \mathbf{u}\|_{1}=\sum_{i=1}^{n} \sqrt{\left(\nabla_{x} \mathbf{u}_{i}\right)^{2}+\left(\nabla_{y} \mathbf{u}_{i}\right)^{2}+\left(\nabla_{z} \mathbf{u}_{i}\right)^{2}}$

where $n$ represents the total number of discrete points (usually pixels of an image). As a result, it is not possible to formulate an explicit gradient operator for the entire discrete field without separately employing directional gradient operators. One popular method is based on anisotropic separable approximation [11]:

$$
\|\nabla \mathbf{u}\|_{1} \approx \mathbf{D u}=\left|\mathbf{D}_{\mathbf{x}} \mathbf{u}\right|+\left|\mathbf{D}_{\mathbf{x}} \mathbf{u}\right|+\left|\mathbf{D}_{\mathbf{z}} \mathbf{u}\right|
$$

where horizontal, vertical and depth discrete derivative operators are denoted by $\mathbf{D}_{\mathbf{x}}, \mathbf{D}_{\mathbf{x}}$ and $\mathbf{D}_{\mathbf{z}}$, respectively, each in the form of a $n \times n$ matrix. Such approximation sacrifices accuracy for the simplicity of numerical maneuver and will introduce large matrix computation and storage during the optimization process (section 2.2). Individual elements in $\mathbf{D}$, while straightforward to calculate in digital images because of the regular grid of pixels, are not trivial to calculate accurately when the nodes of the discrete mesh of $\mathbf{u}$ are distributed irregularly in space. Furthermore, this definition $(4,5)$ can differ substantially from the TV of the underlying continuous field, depending on the resolution of $\mathbf{u}$.

Therefore, we define an alternative approximation of the continuous form of TV as:

$$
T V(\mathbf{u})=\Sigma_{i=1}^{N}\left|\nabla \varphi_{i} \mathbf{u}\right| \approx \int_{\Omega_{h}}|\nabla u| d \Omega_{h}
$$

where a numerical integration is performed over the $3 \mathrm{D}$ myocardial field by $\mathrm{N}$ (at the order of $10^{5}$ ) Gaussian quadrature points. Depending on the discretization method used (meshfree method [21]in this paper), $\nabla u$ on each Gauss point is approximated by a linear combination of its neighboring nodal points in the discrete field $\mathbf{u}$ based on the $3 \times n$ spatial gradients of the shape functions $\varphi_{i}$. Because each Gauss point has only a small set of support nodal points, $\varphi_{i}$ and $\nabla \varphi_{i}$ are sparse with a small number of non-zero values. This definition of $T V(\mathbf{u})(6)$ does not directly rely on the discrete field defined over $\mathbf{u}$, hence it is robust to the spatial resolution of $\mathbf{u}$. Furthermore, it is also consistent with the data-fidelity term in equation (3), where the biophysical model $\mathbf{H}$ is also calculated from numerical approximations of integrals involved in the quasistatic Maxwell equations.

\section{B. Iteratively re-weighted TV minimization (IRTV)}

Once the L1-norm is applied to the constraint term, the corresponding object function (3) become difficult to solve. Here, we adopt the concept of iteratively re-weighted (IR) to handle this challenge. The general idea of IR is based on the following iterative approximation of a general variable $x$ where at iteration $k$ :

$$
\left|x^{(k)}\right| \approx\left\|\frac{x^{(k)}}{\sqrt{x^{(k-1)}}}\right\|_{2}^{2}
$$

namely, at each iteration, the L1-norm of $x$ is approximated by the L2-norm of a weighted- $x$, where the weight is the square root value of the $x$ obtained at the previous iteration. Because the value of $x$ from the previous iteration is known, the $\mathrm{L} 1$ regularization problem can be approximated by a sequence of weighted L2 regularization with the weight changing at each iteration depending on the solution from the previous iteration.

Adopting the concept of IR [15], we can approximate the continuous form of TV as a sequence of L2-norm of weighted $\nabla u$, each weight being the square root of $\nabla u$ at the previous $(k-1)$-th iteration, i.e.:

$$
\int_{\Omega_{h}}\left|\nabla u^{(k)}\right| d \Omega_{h} \approx \int_{\Omega_{h}}\left\|\frac{\nabla u^{(k)}}{\sqrt{\nabla u^{(k-1)}}}\right\|_{2}^{2} d \Omega_{h}
$$

Coupling this with our proposed variational approximation of TV(6), equation (8) can be approximated again by variational approximation in weight L2 form:

$$
\begin{aligned}
& \int_{\Omega_{h}}\left\|\frac{\nabla u^{(k)}}{\sqrt{\nabla u^{(k-1)}}}\right\|_{2}^{2} d \Omega_{h} \approx \int_{\Omega_{h}} \frac{\left|\nabla u^{(k)}\right|^{2}}{\left|\nabla u^{(k-1)}\right|} d \Omega_{h} \\
& \approx \mathbf{u}^{(k)^{T}}\left(\sum_{i=1}^{N} \frac{\left(\nabla \varphi_{i}^{T} \nabla \varphi_{i}\right)}{\left|\nabla \varphi_{i} \mathbf{u}^{(k-1)}\right|}\right) \mathbf{u}^{(k)}=\left\|\mathbf{W} \mathbf{u}^{(k)}\right\|_{2}^{2}
\end{aligned}
$$

where: $\mathbf{W}^{T} \mathbf{W}=\sum_{i=1}^{N} \frac{\left(\nabla \varphi_{i}^{T} \nabla \varphi_{i}\right)}{\left|\nabla \varphi_{i} \mathbf{u}^{(k-1)}\right|}$

In another word, at each iteration, the proposed variational form of TV is now approximated by a weighted L2-norm of $\mathbf{u}$, with the weight matrix $\mathbf{W}^{T} \mathbf{W}$ defined as above. It is important to note that, once a discrete mesh of the heart is constructed with the Gaussian quadrature points established, the shape functions used for calculating $\mathbf{W}^{T} \mathbf{W}$ remain fixed and the only change in $\mathbf{W}^{T} \mathbf{W}$ at each iteration comes from previous $\mathbf{u}$. Therefore, at each iteration, computation of the weight matrix involves only weighting $\mathrm{N}$ pre-stored sparse matrices - one for each Gauss point - by a scalar $\left|\nabla \varphi_{i} \mathbf{u}\right|$ and adding them together.

For comparison, the L1-norm of the gradient field $\|\nabla \mathbf{u}\|_{1}$ (5) can also be approximated by weighted L2-norm with the application of IR concept. The weight matrix $\mathbf{D}^{T} \mathbf{W}_{\mathbf{d}} \mathbf{D}$ is assembled from [15]:

$$
\begin{aligned}
& \mathbf{D}=\left(\begin{array}{lll}
\mathbf{D}_{\mathbf{x}} & \mathbf{D}_{\mathbf{y}} & \mathbf{D}_{\mathbf{z}}
\end{array}\right)^{T} \\
& \mathbf{W}_{\mathbf{d}}=\operatorname{diag}\left(\boldsymbol{\Omega}_{\mathbf{d}}{ }^{(k)} \quad \boldsymbol{\Omega}_{\mathbf{d}}{ }^{(k)} \quad \boldsymbol{\Omega}_{\mathbf{d}}{ }^{(k)}\right) \\
& \boldsymbol{\Omega}_{\mathbf{d}}{ }^{(k)}=\operatorname{diag}\left(\left(\mathbf{D}_{\mathbf{x}} \mathbf{u}^{(k)}\right)^{2}+\left(\mathbf{D}_{\mathbf{y}} \mathbf{u}^{(k)}\right)^{2}+\left(\mathbf{D}_{\mathbf{z}} \mathbf{u}^{(k)}\right)^{2}\right)
\end{aligned}
$$

where the dimension of matrix $\mathbf{D}$ is $3 n \times n$ and the dimension of $\mathbf{W}_{\mathbf{d}}$ is $3 n \times 3 n$. It is evident that using the discrete TV form as defined in (5), the IRTV will involve the computation and storage of high-dimension weight matrices that will substantially increase the computational coat.

\section{IRTV-L2 vs. IRTV-L1}

Recent studies have shown that, when combined with a L1-norm regularization term, an L1-norm data-fidelity shows higher robustness to measurement error as well as 
faster convergence in comparison to an L2-based datafidelity norm [5]. To have a better understanding of the difference between an L1- and L2-norm data-fidelity term in our problem, we will consider both IRTV-L2 and IRTVL1 approaches in this study.

IRTV-L2: First, we considering a common least-square data fidelity term (setting $q$ in (3) equal to 2). Combining with the approximation define in $(9,10)$, the IRTVL2 minimization can be solved by a set of weighted L2minimizations:

$$
\begin{aligned}
& \hat{\mathbf{u}}^{(k)}=\min _{\mathbf{u}}\left\{\left\|\mathbf{H u}^{(k)}-\phi\right\|_{2}^{2}+\lambda^{(k)}\left\|\tilde{\mathbf{W}} \mathbf{u}^{(k)}\right\|_{2}^{2}\right\} \\
& \Rightarrow \hat{\mathbf{u}}^{(k)}=\left(\mathbf{H}^{T} \mathbf{H}+\lambda^{(k)} \tilde{\mathbf{W}}{ }^{T} \tilde{\mathbf{W}}\right)^{-1} \mathbf{H}^{T} \phi
\end{aligned}
$$

where $\tilde{\mathbf{W}}^{T} \tilde{\mathbf{W}}=\sum_{i=1}^{N} \frac{\nabla \varphi_{i}^{T} \nabla \varphi_{i}}{\left|\nabla \varphi_{i} \mathbf{u}^{(k-1)}\right|+\beta}$ and $\lambda^{(k)}$ is the regularization parameter used at iteration $k . \beta$ is a small positive value to reduce numerical errors when $\left|\nabla \varphi_{i} \mathbf{u}^{(k-1)}\right|$ at the $i$-th Gauss point is close to zero.

In this way, by iteratively solving the L2 regularization, the local region with a small spatial gradient (being in the denominator) will generate a large penalty in the current iteration, while a large gradient will be promoted until the final solution exhibits a piecewise smooth pattern with steep gradient. The convergency of the solution of IR to the minimum of objective function (3) was proved in [15]. The equation (11) can be represent as a weight L2 form.

IRTV-L1: Second, we also consider the alternative of a L1-norm data fidelity term (setting $q$ in (3) equal to 1 ). The concept of IR can be extended to this IRTV-L1 model by replace the L1-norm data-fidelity term with a sequence of weighted L2-norm, with the weight matrix at each iteration $k$ defined by $\mathbf{W}_{\mathbf{f}}^{T} \mathbf{W}_{\mathbf{f}}=\operatorname{diag}\left(\left|\mathbf{H} \mathbf{u}^{(k-1)}-\phi\right|\right)$. Again, this IRTV-L1 problem can also be solved as a sequence of weighted L2-minimization:

$$
\begin{aligned}
& \hat{\mathbf{u}}^{(k)}=\min _{\mathbf{u}}\left\{\left\|\mathbf{H} \mathbf{u}^{(k)}-\phi\right\|_{1}^{1}+\lambda^{(k)}\left\|\tilde{\mathbf{W}} \mathbf{u}^{(k)}\right\|_{2}^{2}\right\} \\
& \Rightarrow \hat{\mathbf{u}}^{(k)}=\left(\mathbf{H}^{T} \mathbf{W}_{\mathbf{f}}^{T} \mathbf{W}_{\mathbf{f}} \mathbf{H}+\lambda^{(k)} \tilde{\mathbf{W}}{ }^{T} \tilde{\mathbf{W}}\right)^{-1} \mathbf{H}^{T} \mathbf{W}_{\mathbf{f}}^{T} \mathbf{W}_{\mathbf{f}} \phi
\end{aligned}
$$

For both IRTV-L2 and IRTV-L1, matrix inversion is calculated by Conjugate Gradient method in this study.

\section{Algorithm Summary}

To put the whole picture together, we need to resolve two further issues:

Initialization: While the proposed TV method, because of its underlying sparsity promoting nature, is suitable for overcoming the physically ill-posedness of the reconstruction problem, it needs to be initialized with proper methods that can overcome the mathematical ill-posedness of the problem. For this purpose, any regularization method imposing smoothness constraints is expected to be suitable. Here, a simple 0-order Tikhonov regularization is used for obtaining the initial solution of $\mathbf{u}^{(0)}$.

Regularization parameter: For the initialization with Tikhonov regularization, $\lambda^{(0)}$ is calculated by the L-curve method [7]. After initialization, the iteration repeats until the convergence criterion, i.e., the difference between two successive gradients of solutions is smaller than a pre-defined tolerance. Unfortunately, there is currently no established method for objectively determining regularization parameter in L1-based problems, and most works rely on an empirical and supervised procedure to select an optimal value of $\lambda$ after a large set of experiments. In the proposed IRTV method, because the regularization term in the objective function changes in each iteration, a less supervised approach for the selection of $\lambda^{(k)}$ is desired for a robust and automatic algorithm. Here we adopt the method proposed in [17] to automatically update the magnitude of $\lambda^{(k)}$ at each iteration based on the infinity norm of the matrices involved in the data-fidelity and the regularization terms (See Algorithm 1 the expression of $\lambda^{(k)}$ ).

A complete summary of the algorithm for both the IRTVL2 and IRTV-L1 is provide in Algorithm 1.

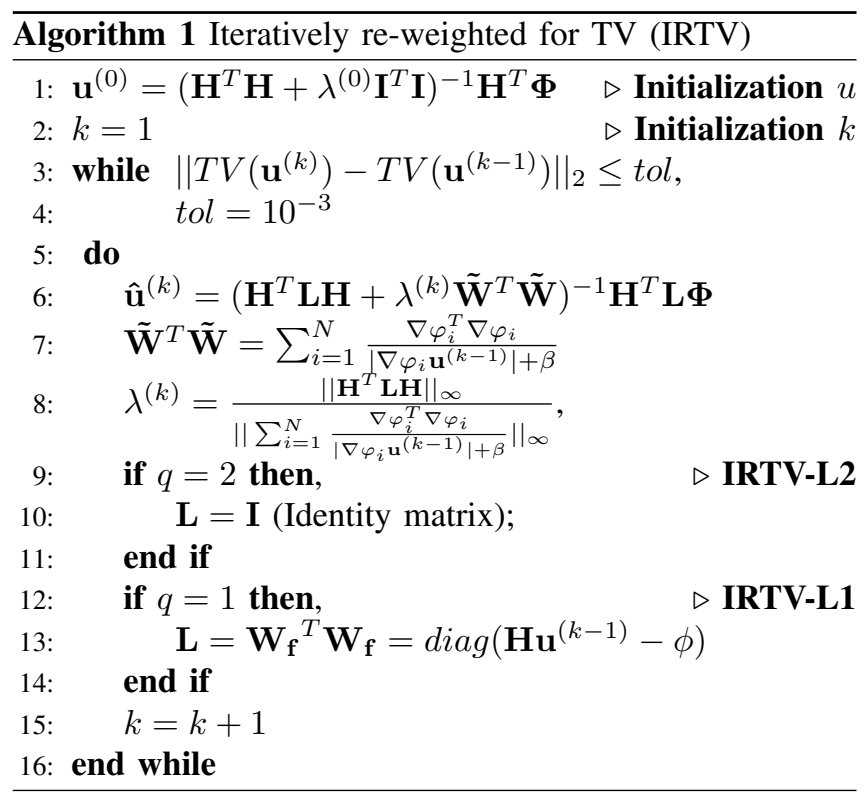

\section{PHANTOM EXPERIMENTS}

First, we evaluate the proposed method through phantom experiments conducted on four realistic human heart-torso models derived from CT scans. We focus on the ability of the proposed method to outline the steep gradient of action potential along the border of ischemic myocardium that separates the region of inactive and active tissue during the ST-segment of an ECG cycle. Ischemic myocardium of different sizes and locations are considered. Throughout all experiments, the accuracy of the proposed method is primarily measured by the consistency metric: CoM $=\frac{S_{1} \cap S_{2}}{S_{1} \cup S_{2}}$ where $S_{1}$ represents region of steep gradients (in terms of the number of meshfree nodes) in the reconstructed action potentials and $S_{2}$ is the region of steep gradients in the 


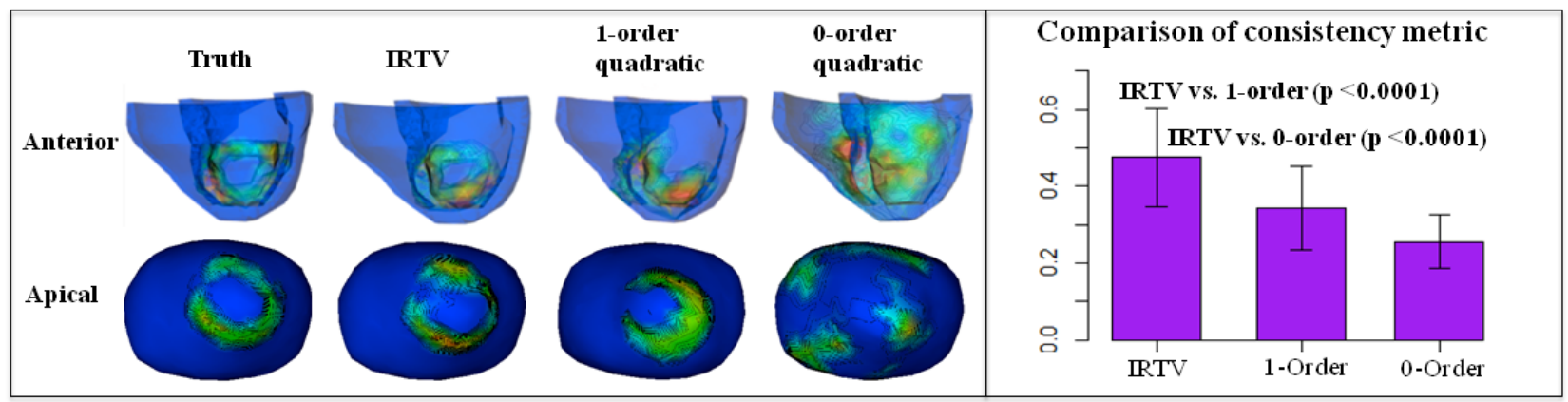

A. Two examples of steep gradient along ischemic border

B. Statistic analysis of 137 cases

Figure 2. Examples (A) and statistic analysis (B) in reconstructing the steep action potentials gradient along ischemic border

Table I

Consistency metric BETWEEN THE RECONSTRUCTED AND TRUE REGIONS OF STEEP ACTION POTENTIAL GRADIENTS, WITH RESPECT TO LOCATIONS (TOP) AND SIZES (BOTTOM) OF THE ISCHEMIC REGION.

\begin{tabular}{|c|c|c|c|c|c|}
\hline Segment/Method & $\begin{array}{c}\text { Anterior } \\
(\mathrm{n}=26)\end{array}$ & $\begin{array}{c}\text { Inferior } \\
(\mathrm{n}=21)\end{array}$ & $\begin{array}{c}\text { Lateral } \\
(\mathrm{n}=40)\end{array}$ & $\begin{array}{c}\text { Septal } \\
(\mathrm{n}=29)\end{array}$ & $\begin{array}{c}\text { Apex } \\
(\mathrm{n}=4)\end{array}$ \\
\hline IRTV(q=2) CoM & $0.5153 \pm 0.10$ & $0.4889 \pm 0.07$ & $0.5375 \pm 0.11$ & $0.3423 \pm 0.09$ & $0.6146 \pm 0.13$ \\
\hline 1-order CoM & $0.3624 \pm 0.07$ & $0.3169 \pm 0.07$ & $0.3995 \pm 0.10$ & $0.2675 \pm 0.12$ & $0.4677 \pm 0.17$ \\
\hline 0-order CoM & $0.2419 \pm 0.06$ & $0.2431 \pm 0.05$ & $0.2909 \pm 0.08$ & $0.2325 \pm 0.07$ & $0.1845 \pm 0.09$ \\
\hline Size/Method & $\begin{array}{c}0 \sim 5 \% \\
(\mathrm{n}=29)\end{array}$ & $\begin{array}{c}5 \% \sim 10 \% \\
(\mathrm{n}=31)\end{array}$ & $\begin{array}{c}10 \% \sim 20 \% \\
(\mathrm{n}=49)\end{array}$ & $\begin{array}{c}\geq 20 \% \\
(\mathrm{n}=28)\end{array}$ & $\begin{array}{c}\text { Total } \\
(\mathrm{n}=137)\end{array}$ \\
\hline IRTV (q=2) & $0.5134 \pm 0.10$ & $0.4739 \pm 0.14$ & $0.4775 \pm 0.13$ & $0.4281 \pm 0.11$ & $0.4742 \pm 0.13$ \\
\hline 1-order & $0.3268 \pm 0.12$ & $0.3383 \pm 0.11$ & $0.3524 \pm 0.11$ & $0.3502 \pm 0.09$ & $0.3433 \pm 0.11$ \\
\hline 0-order & $0.2225 \pm 0.07$ & $0.2706 \pm 0.09$ & $0.2643 \pm 0.07$ & $0.2552 \pm 0.04$ & $0.2551 \pm 0.07$ \\
\hline
\end{tabular}

ground truth. In the current study, region of steep gradients is outlined using a threshold value that is automatically calculated from the mean and standard deviation of the calculated gradients. According to electrophysiology, action potentials during the ST-segment are set to be $-20 \mathrm{mV}$ for the ischemia region, and $20 \mathrm{mV}$ for the health region [13]. 370-lead body-surface ECG are simulated and corrupted with Gaussian noise as inputs.

\section{A. IRTV vs. Existing Quadratic-regularization:}

Here, we consider 137 cases of ischemic region with different locations and with size ranging from $0.5 \%$ to $50 \%$ of LV. On average the IRTV takes 26 iterations to converge.

Fig. 2A shows two examples of ground truth where the steep spatial gradients of action potentials is distributed along the border of ischemic myocardium, respectively, located at anterior and apical regions of the LV. This spatial structure of the steep gradient is well preserved in action potentials reconstructed by the presented IRTV-L2 $(q=2)$ method. In comparison, gradient of the action potential reconstructed by the 0 -order quadratic method is diffused and does not reveal the location or the shape of the underlying ischemic region. The 1-order quadratic regularization shows improved accuracy over its 0-order counterpart but the reconstructed gradient is still blurred and loses the structure/topolgy of the ischemic border. Fig. $2 \mathrm{~B}$ lists the consistency metric for results obtained on all
137 cases by the three methods, where paired student's $t$ test shows that the accuracy of IRTV is significantly higher than the other two method based on quadratic regularization $(p<0.0001)$.

In addition, Table 1 lists the consistency metric of all results with respect to the locations and sizes of the ischemic region. Student's t-tests and one-way ANOVA tests are used to compare how the results from IRTV differ, respectively, between all size groups and all location groups, except that of apical region. As shown, it is more difficult to correctly capture the gradient of action potentials using IRTV when ischemia happens at the septal region of the LV $(0.3423 \pm 0.09)$ compared to the anterior region $(0.5153 \pm 0.1$, $p<0.0001$ ), inferior region ( $0.4889 \pm 0.07, p<0.0001$ ), or lateral region $(0.5375 \pm 0.11, p<0.0001)$ of the LV. This is because the septum is most hidden from body-surface observations [23]. In comparison, there is no significant difference in the accuracy of IRTV (ANOVA $p=0.19$ ) in outlining the gradient along ischemic region of different sizes.

\section{B. Discrete TV-prior vs Variational TV-prior}

A set of 72 studies are conducted to compare the performance of IRTV-L2 using the proposed variational definination (9) versus traditional discrete definition of TV (10). During the IR algorithm, the difference between these two TV definitions is exhibited as the way the re-weighting 


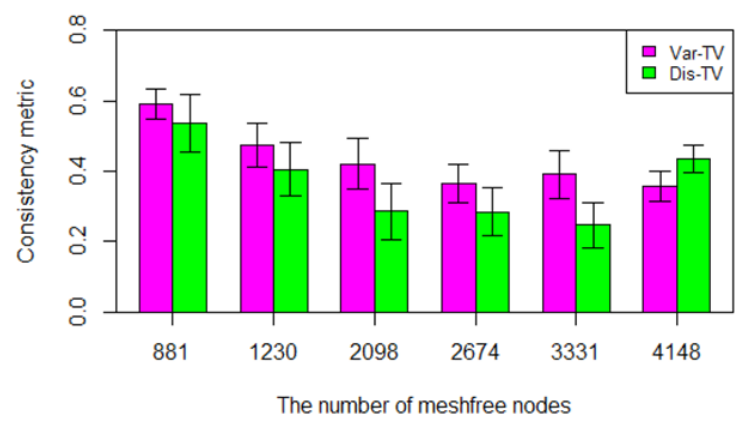

(a) Accuracy of Discrete TV and Variational TV

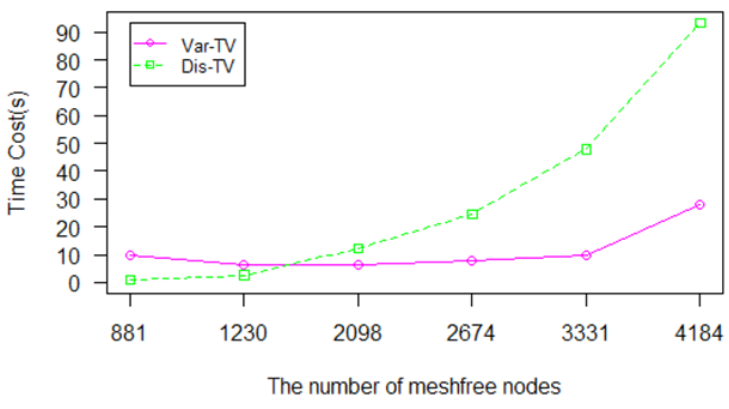

(b) Time consumption of Discrete TV and Variational TV

Figure 3. Comparison study between the discrete TV and variational TV: (a) the accuracy in preserving the steep gradient along ischemic border based on two different TV prior. (b) time consumption of each method under the same operation system.

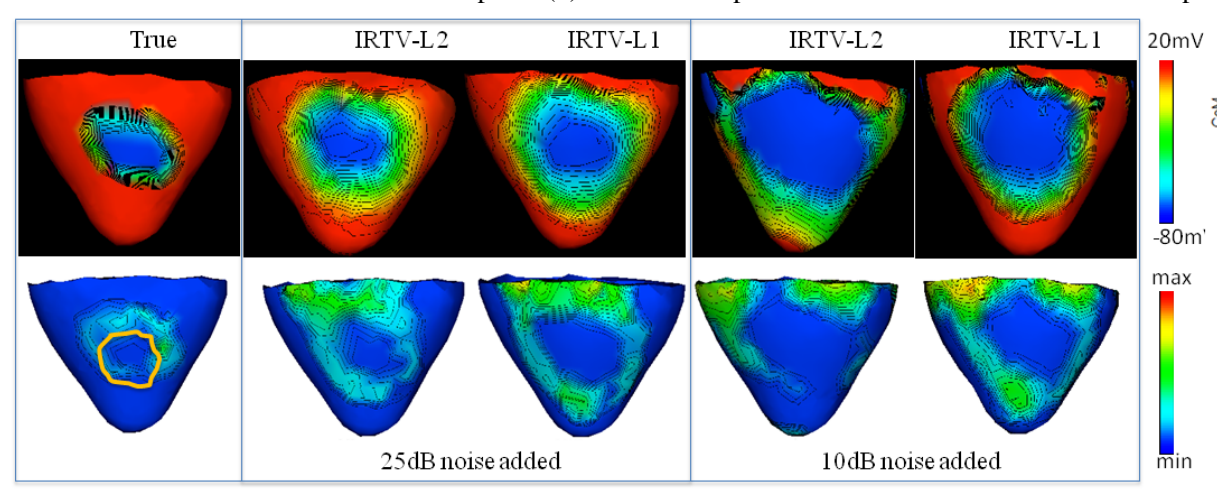

(a) Reconstruction of (top) action potential and (bottom) steep gradient along the ischemic region

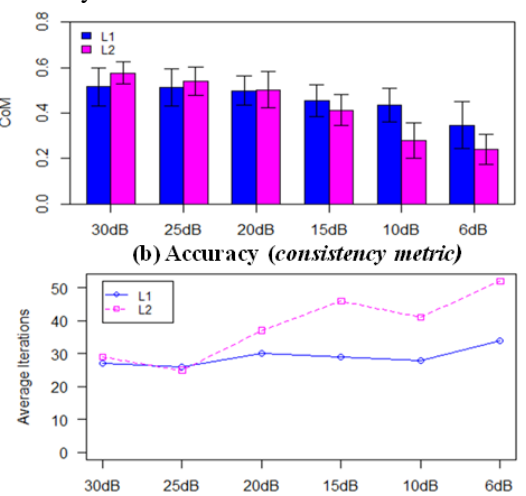

(c) Iteration time of IRTV-L1 and IRTV-L2

Figure 4. IRTV-L1 and IRTV-L2 reconstruction analysis. Examples (a) at 25dB and 10dB noise. The color bars indicate the value of action potential (top) and its gradient (bottom) change,respectively. The accuracy (b) and average iterations (c) at different noises.

matrix is calculated in each iteration: $\mathbf{W}^{T} \mathbf{W}$ as defined in (6) for variational $\mathrm{TV}$, versus $\mathbf{D}^{T} \mathbf{W}_{\mathbf{d}} \mathbf{D}$ in $\sqrt{10}$ for discrete TV.

Fig 3 (a) shows the accuracy (consistency metric) of discrete TV and variational TV in preserving the steep gradient under different mesh resolution $(3 \sim 6 \mathrm{~mm})$. As shown, variational TV delivers a more consistent accuracy among different resolution than discrete $\mathrm{TV}$, demonstrating a higher robustness to mesh resolution as hypothesized.

Fig 3 (b) shows the averaged computation cost per iteration for minimizing (3) using variational TV and discrete TV under different mesh resolution (total number of iterations for average are similar). The computation time is reported using MATLAB with Dual 2.66-GJz Intel cores. As the mesh resolution increases, the discrete $\mathrm{TV}$ shows a substantially increasing demand in computation time as the dimension of the directional gradient operator $\mathbf{D}$ in (10) increases dramatically. In comparison, the computation cost of variational remains stable, little affected by the mesh resolution and substantially lower than that of discrete TV for a dense mesh.

\section{IRTV-L2 VS IRTV-L1}

The comparison study between IRTV-L2 and IRTV-L1 is conducted on 15 cases for each different levels of signal-to- noise ratio $(6 \mathrm{~dB}, 10 \mathrm{~dB}, 15 \mathrm{~dB}, 20 \mathrm{~dB} 25 \mathrm{~dB}, 30 \mathrm{~dB})$ Gaussian noises added on body-surface ECGs and in total 90 cases for each method.

Fig 4 (b) shows the accuracy (consistency metric) of IRTV-L1 and IRTV-L2 in preserving the steep gradient of reconstructed action potential under different noise levels. As shown, IRTV-L1 is more robust to large measurement noises, while IRTV-L2 sees a much faster deterioration of accuracy as the measurement noise increases. As an example, Fig 4 (a) compares the results of IRTV-L2 and IRTV-L1 in reconstructing the spatial distribution of action potential and preserving its steep spatial gradient along the border of an ischemic region at anterior $\mathrm{LV}$. With $25 \mathrm{~dB}$ measurement noises, the spatial structure of the steep gradient is well preserved by both IRTV-L2 and IRTV-L1, although IRTVL2 shows a higher consistency with the ground truth. When noise level is increased (i.e. 10dB), the performance of IRTV-L2 drastically decreased while the IRTV-L1 is still able to preserve the structure of the ischemic border with reasonable accuracy.

Fig 4 (c) shows the averaged convergence speed (in terms of the number of iterations taken to convergence) of the two methods at different noise levels. As shown, IRTV-L1 takes 


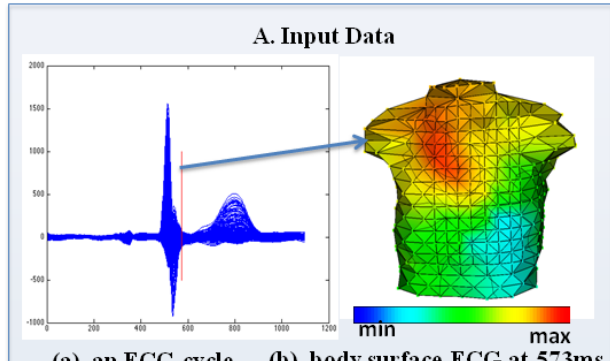

(a) an ECG cycle (b) body surface ECG at $573 \mathrm{~ms}$

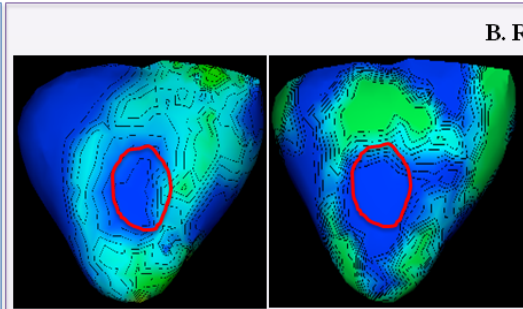

(a) IRTV-L2
B. Result

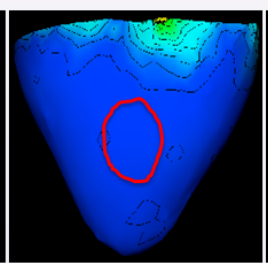

(b) IRTV-L1

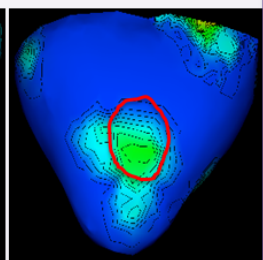

(c) 1-order

(d) 0-order

Figure 5. Input data (A) and result (B) of case 1. Input data contains (A.a) an ECG cycle and the red line labels the selected time frame (573 ms) of body surface mapping in (A.b). Result shows spatial gradients of 3D action potentials reconstructed from IRTV-L1 and -L2 and quadratic methods on post-infarction human hearts. The red cycles represent the core of the MRI-delineated infarcts.

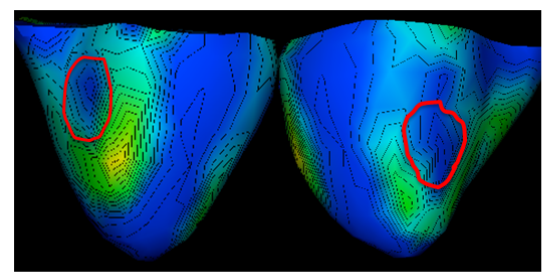

(a) IRTV-L2

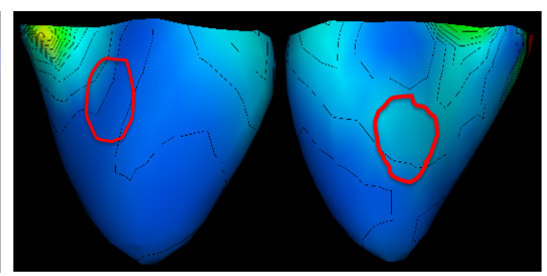

(b) IRTV-L1

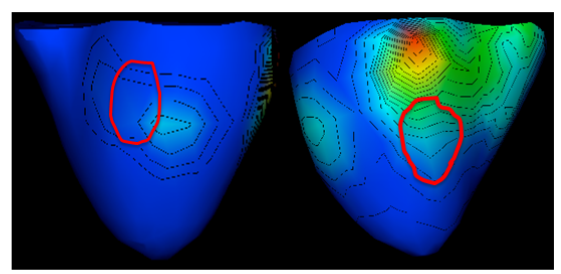

(c) 0-order

Figure 6. Results of case 2, which include the IRTV-L2 and -L1 and 0-order quadratic method.

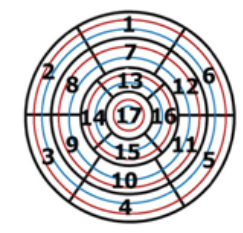

Figure 7. AHA segment

Table 2. Infarct quantification results compared with provided Gold Standard(GS)

\begin{tabular}{|l|c|c|c|c|}
\hline & & Center & Location & Segment Overlap (SO) \\
\hline Case 1 & $G S$ & $10 / 11$ & $3,4,5,9,10,11,12,15,16$ & NA \\
& Results & $10 / 15$ & $3,9,10,11,12,14,15,17$ & $66.7 \%$ \\
\hline Case 2 & $G S$ & 15 & $1,9,10,11,15,17$ & NA \\
& Results & 15 & $1,7,9,10,11,15,17$ & $71.4 \%$ \\
\hline
\end{tabular}

a similar number of iterations to converge in the presence of different measurement noises, while IRTV-L2 takes longer to converge as the noise level increases. As a result, with moderate to high level of measurement noises, IRTV-L1 shows faster convergence than IRTV-L2. Nevertheless, under the same computing environment, IRTV-L1 engages slightly more computation time $(12.07 \mathrm{~s})$ per iteration compare to IRTV-L2 (11.03s).

\section{HUMAN STUdY}

Real-data experiments are further performed on two ischemic patients with MRI and body-surface ECG data made available to this study by the 2007 PhysioNet / Computers in Cardiology Challenges [6]. Cardiac MRI data are used to construct the patient-specific heart-torso model. Bodysurface ECGs were recorded by Dalhousie University protocol [18]; each body-surface ECG recording (Fig $5 \mathrm{~A}(\mathrm{a})$ ) consists of a single QRST complex and we select a time frame within the ST interval as input. Fig 5 A(b) illustrate the selected input body-surface potential map at $573 \mathrm{~ms}$ for case 1. Gold standards of infarct quantification were obtained form LGE MRI by cardiologists blinded to this study.
Unlike phantom experiments, the gold standards provided quantify the location and size of the infarct according to the AHA 17-segment model of LV [3] (Fig.7), revealing core regions of the infarct as labeled by the red cycle in Fig.5.

The patient of case 1 has one ischemic region with its core located at middle septal-inferior LV (segment 9 and segment 10). As shown in Fig 5 spatial distribution of action potential reconstructed by IRTV-L2 exhibits a steep gradient that are localized and distributed along ischemic core. Similar result is obtained with IRTV-L1. In comparison, action potential reconstructed from neither of the other two quadratic methods reveal any physiological meaningful information regarding the existence, location, or structure of the ischemic myocardium.

The patient of case 2 has two separated ischemic regions (Fig 6), one at basal-anterior of LV (segment 1) and the other at apical-inferior of LV (segment 15). In existing works, this has been shown to pose additional challenges on ischemic region detection [20]. As shown in Fig 6, the spatial gradient of action potential reconstructed by IRTV-L2 are correctly localized around both ischemic region, revealing the location and extent of both ischemic cores. IRTV-L1, in comparison, 
partially misses the localization of one ischemic region. 0 -order quadratic method only reveals certain information in inferior ventricular wall (right) without revealing any physiological meaningful structure, and it misses all the information in anterior wall (left). 1-order quadratic method fails to coverage under all the possible parameters using the cvx software mentioned in [19]. These observations in real data study are consistent with the findings in our phantom experiments.

Table 2 lists quantification comparison with the gold standard. As shown, the ischemic centers are correctly identified in both patients and the accuracy is comparable to the best result available [20]. In particular, in case 2, our method shows much higher accuracy in localizing the two separated ischemic region with $S O=71.4 \%$, while the best available $S O=33.33 \%$ in literature [20]).

\section{CONCLUSION}

This paper presents a novel approach to reconstruct 3D ischemic region based on a new variational TV-prior, physiologically motivated by the unique spatial property of electrophysiology of the heart that is in line with the role of TV penalty. In comparison, existing works using TV-prior for epicardium potential reconstruction [5] are restricted to the heart surface and are not tied to the unique electric property of ischemic region, more important, the present work can extend TV-minimization to a border range of application that involve physical domain of complex shape and unstructured volumetric mesh. In the future, we will investigate the incorporation of temporal constraints into proposed algorithm.

\section{ACKNOWLEDGEMENT}

This work is in part supported by the National Science Foundation CAREER Award ACI-1350374.

\section{REFERENCES}

[1] R. J. Bing. Myocardial ischemia and infarction: growth of ideas. Cardiovascular research, 51(1):13-20, 2001.

[2] J. E. Burnes, B. Taccardi, R. S. MacLeod, and Y. Rudy. Noninvasive ecg imaging of electrophysiologically abnormal substrates in infarcted hearts a model study. Circulation, 101(5):533-540, 2000.

[3] M. D. Cerqueira, N. J. Weissman, et al. Standardized myocardial segmentation and nomenclature for tomographic imaging of the heart. Circulation, 105(4):539-542, 2002.

[4] T. Chan and C. K. Wong. Total variation blind deconvolution. IEEE Trans. Image Process., 7(3):370-375, 1998.

[5] S. Ghosh and Y. Rudy. Application of L1-norm regularization to epicardial potential solutions of the inverse electrocardiography problem. Ann. Biomed. Eng., 37(5):902-912, 2009.

[6] A. L. Goldberger et al. Physiobank, physiotoolkit, and physionet: Components of a new research resource for complex physiological signals. Circulation, 101:e215-e220, 2000.

[7] P. Hansen and D. O'Leary. The use of the l-curve in the regularization of discrete ill-posed problems. SIAM Journal on Scientific Computing, 14(6):1487-1503, 1993.
[8] R. Jennings, C. Steenbergen Jr, and K. Reimer. Myocardial ischemia and reperfusion. Monographs in pathology, 37:4780, 1994.

[9] Y. Jiang, C. Qian, R. Hanna, D. Farina, and O. Dössel. Optimization of the electrode positions of multichannel ecg for the reconstruction of ischemic areas by solving the inverse electrocardiographic problem. International Journal of Bioelectromagnetism, 11(1):27-37, 2009.

[10] G. Li and B. He. Non-invasive estimation of myocardial infarction by means of a heart-model-based imaging approach: a simulation study. Med. Biomed. Eng. Com., 42(1):128-136, 2004.

[11] Y. Li and F. Santosa. A computational algorithm for minimizing total variation in image restoration. IEEE Trans. Image Process., 5(6):987-995, 1996.

[12] M. C. MacLachlan, B. F. Nielsen, M. Lysaker, and A. Tveito. Computing the size and location of myocardial ischemia using measurements of st-segment shift. IEEE Trans. Biomed. Eng., 53(6): 1024-1031, 2006.

[13] B. F. Nielsen, M. Lysaker, and P. Grøttum. Computing ischemic regions in the heart with the bidomain model: First steps towards validation. IEEE Trans. Med. Imag., 32(6):1085-1096, 2013.

[14] R. Plonsey. Bioelectric Phenomena. Wiley Online Library, 1999.

[15] P. Rodríguez and B. Wohlberg. Efficient minimization method for a generalized total variation functional. IEEE Trans. Image Process., 18(2):322-332, 2009.

[16] L. I. Rudin, S. Osher, and E. Fatemi. Nonlinear total variation based noise removal algorithms. Phys. D: Nonlinear Phenom., 60(1):259-268, 1992.

[17] M. Schmidt, G. Fung, and R. Rosales. Optimization methods for 11-regularization. University of British Columbia, Technical Report TR-2009-19, 2009.

[18] L. M. Title, S. E. Iles, M. J. Gardner, C. J. Penney, J. C. Clements, and B. M. Horáček. Quantitative assessment of myocardial ischemia by electrocardiographic and scintigraphic imaging. J. Electrocardiol., 36:17-26, 2003.

[19] D. Wang, R. M. Kirby, R. S. MacLeod, and C. R. Johnson. Identifying myocardial ischemia by inversely computing transmembrane potentials from body-surface potential maps. In Int. Conf. Bioelectromagnetism, pages $121-125$, Salt Lake, UT, 2011.

[20] L. Wang, K. C. Wong, H. Zhang, H. Liu, and P. Shi. Noninvasive computational imaging of cardiac electrophysiology for 3-D infarct. IEEE Trans. Biomed. Eng., 58(4):1033-1043, 2011.

[21] L. Wang, H. Zhang, K. C. Wong, H. Liu, and P. Shi. Noninvasive volumetric imaging of cardiac electrophysiology. In IEEE conf. Computer Vision and Pattern Recognition (CVPR), pages 2176-2183. IEEE, 2009.

[22] K. Wasserman, J. E. Hansen, D. Y. Sue, W. W. Stringer, and B. J. Whipp. Principles of exercise testing and interpretation: including pathophysiology and clinical applications. Lippincott Williams \& Wilkins Philadelphia, 2005.

[23] J. Xu, A. R. Dehaghani, F. Gao, and L. Wang. Localization of sparse transmural excitation stimuli from surface mapping. In Med. Image. Comput. Comput. Assist. Interv. (MICCAI), pages 675-682. Springer, 2012. 